\title{
Classifiers in Event and Nominal Plurality in Mandarin Kyumin $\mathrm{Kim}^{\dagger}$ \\ Chungbuk National University
}

\begin{abstract}
Event plurality indicates multiplicity of an event. One of the major issues pertaining to event plurality in the literature has been the extent to which it is parallel to nominal plurality. The current literature suggests that event and nominal plurality are in parallel in that a plural event denotes a mass meaning similar to a bare plural noun that denotes mass. However, a plural event in some languages such as Mandarin does not show a mass meaning, and a bare plural noun is lacking in the language. This paper addresses a question of how to characterize a parallel between event and nominal plurality in Mandarin. It provides a novel proposal in which the parallel in Mandarin can be characterized in terms of corresponding classifiers in the event and nominal domains. A major contribution of this paper is that it suggests that event plurality is not a unified phenomenon similar to nominal plurality.
\end{abstract}

Keywords: event, nominal, plural, classifier, parallel

\section{Introduction}

Event plurality indicates multiplicity of an event described by the verb phrase or multiplicity of an occasion in which an event has taken place (e.g., Cusic, 1981; Lasersohn, 1995; Corbett, 2000). An instance of event plurality is illustrated in (1) from Kaqchikel, a Mayan language. ${ }^{1)}$ In (1a), the event of my sitting is described by the verb phrase, which is a simple event without pluralization. This event can be pluralized by being marked by the verbal suffix $-(u) l o ̈ j$, as illustrated in (1b). ${ }^{2)}$ A pluralized event such as in (1b) indicates that the event took place multiple times.

1) All cited data in this paper is presented exactly as given in the source reference. Abbreviations are as follows: $1=$ first person, $\mathrm{A}=$ absolutive, $\mathrm{CLn}=$ nominal classifier, $\mathrm{CLv}=$ verbal classifier, $\mathrm{COM}$ = Completive Aspect, $\mathrm{E}=$ ergative, $\mathrm{PERF}=$ perfect, P.ITV=Positional Intransitive, PLR = pluractional, WP $=$ Witnessed Past (indicates a perfective form of the verb in Chechen).

2) This type of a morpheme is referred as a pluractional morpheme (Newman 1980). As this term is not relevant to the main argumentation of this paper, it is not used in this paper.

† Corresponding author: kukim@chungbuk.ac.kr

Copyright (c) 2021 Language Education Institute, Seoul National University.

This is an Open Access article under CC BY-NC License (http://creative-commons.org/licenses/by-nc/4.0). 
(1) a. X-i-tzuy-e'.

COM-A1s-sit-P.ITV

'I sat.'

b. X-i-tzuy-ulöj.

COM-A1s-sit-ulöj

'I sat many times.' $\quad$ (Henderson 2013)

In a language such as Mandarin, an event can be pluralized via a verbal classifier, as illustrated in (2).3) The verb phrase in (2) describes an event of coughing, and the presence of the verbal classifier xia indicates that the event happened multiple times. In particular, the event took place three times as indicated by the numeral san 'three', which is obligatory in the presence of a verbal classifier.

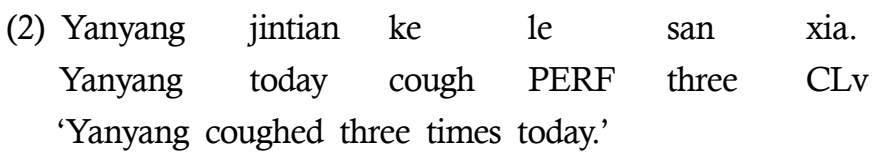

One of the main issues addressed in the literature on event plurality is what parallel can be drawn between event and nominal plurality. For instance, it was proposed that a plural event denotes mass in parallel to a plural noun which also denotes mass (e.g., Yu, 2003; Henderson, 2013). However, it is questionable what parallel can be drawn in a language such as Mandarin that does not have regular nominal plural marker (e.g., Iljic, 2001; Kim and Melchin, 2018). It appears that the parallel in a Mandarin type language cannot be characterized in terms of mass denotation. This paper addresses the question of how to characterize the parallel between event and nominal plurality in a language without a regular plural marker on a verb or a nominal, by examining Mandarin as an empirical domain. By noting that event plurality in the language is expressed by different types of verbal classifiers similar to the nominal plurality expressed by different types of nominal classifiers, this paper provides a novel proposal in which the different verbal classifiers are in a parallel to the corresponding types of nominal classifiers in terms of semantic selection relation and having semantically similar unit for counting. Building on this result, it is further proposed that both event and nominal plurality in Mandarin can

3) Mandarin data without citation is from consultation with four native Mandarin speakers. 
be characterized in terms of the corresponding classifiers in the verbal and nominal domains. ${ }^{4}$

The rest of this paper is organized as follows. Section 2 discusses the previous approaches to a parallel between event and nominal plurality in terms of mass meaning, and shows that event plurality in Mandarin and its parallel to nominal plurality cannot be accounted for by such approaches. Section 3 proposes that there is a certain parallel between verbal and nominal classifiers in Mandarin with respect to some semantic properties of those classifiers, and how the parallel between event and nominal plurality in the language can be understood in terms of the classifiers. Section 4 presents the syntactic consequences of the parallel between verbal and nominal classifiers. Section 5 concludes the paper.

\section{Parallel between Event and Nominal Plurality}

A plural event has been proposed to yield a mass reading similar to mass denotation of a noun which is suggested to be universal (Yu, 2003). Central to this proposal is the fact that a plural event cannot be counted via a numeral just like a plural noun whose denotation is mass. Non-countability of a plural event is exemplified by Chechen, a Nakh language, in (3). In (3a), the event of drinking tea is pluralized: the event took place multiple times. Event plurality is indicated by the vowel alternation on the verb (see Yu, 2003 for detail). The plural event in (3a) is counted as shown in (3b) by the numeral adverbial yttaza 'ten times' and the sentence becomes ungrammatical.

(3) a. adama takhan duqqa'a chai miilira Adam.ERG today many tea drink.PLR.WP

'Adam drank a lot of tea over and over again today.'
b. *adama
takhan
yttaza
chai
miillira
Adam.ERG today ten times tea
drink.PLR.WP
'Adam drank tea ten times today.'
(Yu 2003)

4) This cannot be generalized to other classifier languages such as Korean or Japanese that does not have a verbal classifier to pluralize an event. I do not question how a parallel between event and nominal plurality can be characterized in such languages, which is beyond the scope of this paper. 
In the literature on event plurality, one of the major theoretical assumptions is that there is a parallel between event and nominal domains (e.g., Bach, 1986; Jackendoff, 1991). Under this view, for example, there must be a certain parallel between event and nominal plurality. As mentioned earlier, for example, event plurality is in parallel to nominal plurality in that it yields mass meaning (cf. Yu, 2003; Henderson, 2013).

Although significant, this proposal does not straightforwardly apply to a Mandarin type language. Mandarin pluralizes an event or an occasion by means of a verbal classifier (e.g., Zhu, 1982; Chao, 1986; Deng, 2013; Zhang, 2017). An example of event pluralization is illustrated in (4) (pluralization of an occasion will be detailed in section 3). In (4), an event of Yanyang's jumping is expressed, and this is pluralized by the verbal classifier (CLv) xia. With the accompanying numeral shi 'ten', the CLv indicates that the event of Yanyang's jumping happened ten times. In this paper, the type of a CLv illustrated in (4) is referred as an event CLv (while the type of a CLv that pluralizes an occasion will be referred as an occasion CLv, which will be detailed in section 3.1).5)

(4) Yanyang tiao-le shi $\quad$ xia.
Yanyang jump-PRF ten
'Yanyang jumped ten times.'

The fact that the number of an event can be counted as shown in (4) suggests that event plurality in Mandarin cannot be described in terms of a mass meaning. Rather, a plural event in the language seems to be countable. In fact, it has been proposed that the role of CLvs (as well as nominal classifiers) is more specific than a pluralizer of an event that appears on the verb found in other languages such as Chechen (see (3a)) or Kaqchikel (see (1b)): a CLv has a role of counting (e.g., Deng, 2012; Zhang, 2017; Kim, 2019). Considering the fact that a nominal in Mandarin is also countable by a nominal classifier (as will be detailed in section 3), event and nominal plurality in Mandarin seem to be in parallel by being countable, as recent studies suggest (e.g., Deng, 2013; Kim, 2019). However, this view of counting is rather simplified, and does not provide an account for what

5) Different names are used for different verbal classifiers. In Zhang (2017), for instance, an event CLv is termed as an internal CLv and an occasion CLv is termed as an external CLv. However, I refer them as an event and an occasion CLv for ease of exposition. 
is specifically in parallel between a plural event and a plural nominal in the language beyond counting. For example, in a language with a mass reading of a plural event such as Kaqchikel shown in (1), a more specific parallel between event and nominal plurality than the parallel in terms of mass meaning as in $\mathrm{Yu}$ (2003) has been proposed: a plural event in the language is in parallel to a bare plural of a count noun (Henderson, 2013).

Unlike an English type language, Mandarin has no regular nominal plural marker; thus, a bare plural noun is also lacking (e.g., Iljic, 2001; Kim and Melchin, 2018),6) and the language is well known to be absent with a count-mass distinction (Chierchia, 1998; Borer, 2005). Furthermore, nominal plurality is expressed by means of a nominal classifier (CLn) similarly to event plurality just discussed. Thus, an account proposed for Kaqchikel would not be easily transferrable to the Mandarin case under discussion. This paper investigates what specific parallel between event and nominal plurality can be drawn in a language such as in Mandarin where a CL is a means to pluralize both an event and a nominal. This paper addresses this issue by comparing certain semantic properties of a CLv and a CLn in each domain. Building on the fact that Mandarin has several different types of a CLn, it is questionable which types of CLns correspond to the two types of CLvs, the consequence of which will feed into addressing the question of how to characterize a parallel between the event and nominal plurality in Mandarin beyond counting. Although a great deal of research has been conducted on CLns in the language (e.g., Chao, 1968; Li and Thompson, 1981; Tang, 1990; Cheng and Sybesma, 1998; Zhang, 2013), relatively less attention has been paid to CLvs. In particular, there have been no studies that compare CLs in each domain from a point of view of the parallel between event and nominal plurality as in this paper.

I show that the two types of CLvs - an event and an occasion CLv - correspond to a different type of a CLn respectively with respect to two properties, i.e., semantic selection relation and the unit of counting. Specifically, I show that an event CLv corresponds to an individual CLn, while an occasion CLv corresponds to a container measure CLn. Consequently, an event pluralized via an event CLv is in parallel to a noun pluralized by an individual CLn, while an event pluralized by an occasion event CLv is in parallel to a noun pluralized by a container measure CLn.

6) In these studies, it is shown that a plural suffix men in the language has certain properties that cannot be treated as a regular plural marker. For the purpose of the paper, I do not replicate them but refer readers to those studies. 


\section{Parallel between Event and Nominal Plurality}

\subsection{Assumptions and pervious researches on CLs}

It has long been observed that across languages CLns including measure words are employed in counting, and indicate the unit that counts as one (e.g., Greenberg, 1972; Allan, 1977; Croft, 1994). A similar view has been taken for CLns in Mandarin and measure words in the language are also treated as CLns (e.g., Chao, 1968; Li and Thompson, 1981; Cheng and Sybesma, 1998; Wu and Bodomo, 2009; Zhang, 2013). As with these studies, this paper assumes that CLns and measure words in Mandarin can be uniformly treated, and they indicate the unit of counting. A recent study in Mandarin identified seven types of CLns (Zhang, 2013). Among the seven types, two kinds of CLn are of interest in this paper: an individual CLn and a container measure CLn which will be further detailed in the following sections.7)

As mentioned earlier, two types of the CLvs have been identified in Mandarin literature: an event and an occasion CLv. An event CLv pluralizes an event denoted by the verb phrase as shown in (2) repeated as (5a) below. In (5a), the CLv xia pluralizes the event of Yanyang's jumping which took place ten times. The same event in (5a) can be pluralized by an occasion CLv such as hui as illustrated in (5b). Unlike an event CLv, however, an occasion CLv such as hui in (5b) pluralizes an occasion in which the event took place. In (5b), for instance, there were two occasions in which the event of Yanynag's jumping took place: one occasion happened yesterday and the other happened today (see (6) for detail). The number of the event in each of the occasions is not known, unlike the pluralization of the event shown by an event CLv such as in (5a).

(5) a.

$\begin{array}{lll}\text { a. Yanyang tiao-le } & \text { shi } & \text { xia. } \\ \text { Yanyang jump-PRF ten } & \text { CLv } \\ \text { 'Yanyang jumped ten times.' } & \end{array}$

b. Yanyang tiao-le liang hui. Yanyang jump-PRF two CLv

'Yanyang jumped in two occasions.'

7) The names of CLns are from Zhang (2013). Other names are used to refer to the same types of CLns in Mandarin literature; for example, an individual CLn is referred as an individual measure CLn in Chao (1968). Specific names of CLn do not hinge on the main argument of this paper. 
(e.g., one yesterday and the other today)

Event plurality of an occasion was first identified as belonging to event plurality in Cusic (1981), which has been widely acknowledged in literature (e.g., Cabredo Hofherr, 2010; Wood, 2007). In influential study on event plurality in Cusic (1981), it was proposed that event plurality can be understood in two senses. One is to pluralize an individual event as shown with an event CLv such as in (5a). The other is to pluralize an occasion which is loosely defined as a contextually defined time frame in which an event took place. In recent studies on event plurality (e.g., Cabredo Hofherr, 2010), an occasion identified in Cusic (1981) is viewed as a level where an event is temporally disjointed.8) I propose that this is also the level indicated by an occasion CLv in Mandarin supported by data such as in (6) which elaborates the data in (5b). Each of the occasions in which Yanyang's jumping happened is temporally disjointed: one happened yesterday, and another happened today.

(6) Yanyang tiao-le liang hui.

Yanyang jump-PERF two CLv

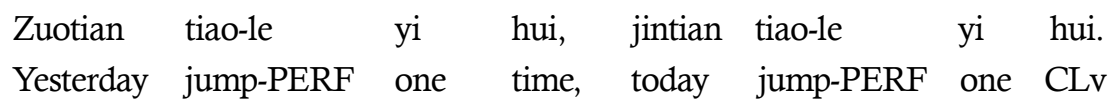

'Yanyang jumped in two occasions. One occasion happened yesterday, and another occasion happened today.'

This paper assumes a widely accepted view on the classification of event in Verkuyl (1972), Vendler (1989), and Smith (1991) among others. Specifically five classes of event will be relevant to the discussion in this paper: accomplishment, achievement, semelfactive, activity, and state. As will be shown in the following sections, a different event CLv can appear with a different event class.

\subsection{Parallel between event CLv and individual CLn}

In this section, I demonstrate that an event CLv is in parallel to an individual

8) An anonymous reviewer questioned if the notion of an occasion can be treated as situation in event semantics. It is not clear whether or not they refer to the same concept, but I leave this issue for further research which is the beyond the scope of this paper. 
CLn with two semantic properties: they show a similar semantic selection relation with an event and a noun that they appear with, and they take a similar unit of counting which is semantically described.

It has been well noted in the literature that there is a semantic selection relation between a CLn and a noun that it appears with (e.g., Chao, 1968; Wu and Bodomo, 2009). For example, consider an individual CLn illustrated in (7). In (7a), the CLn, $z h i$, can appear with a noun such as $b i$ 'pen', but it cannot appear with a noun such as you 'oil' as shown in (7b). What this contrast suggests is that a CLn such as $z h i$ shows a semantic selection relation with a noun that it appears with: it can appear with a noun that is atomic (e.g., a count noun, 'pen' in (7a)) but not with a non-atomic noun (e.g., a mass noun, 'oil' in (7b)). A noun that is atomic has a natural discrete minimal part, in contrast to a non-atomic noun that has no such minimal part (e.g., Links, 1981).9) This type of a CLn that appears with a nominal that is atomic is referred as an individual CLn.

(7) a. san zhi bi

three CLn pen

'three pens'

b. *san zhi you

three CL oil

Intended meaning: 'three drops of oil'

With a numeral, an individual CLn counts the number of an atomic individual denoted by the noun that it appears with. For instance, zhi in (7) counts the number of atomic entity denoted by the noun $b i$ 'pen', but it cannot count the number of non-atomic entity indicated by the noun such as you 'oil'. For an individual CLn, thus, its unit of counting is an atomic entity denoted by the noun that it appears with.

An event CLv shows a semantic selection relation similar to an individual CLn. It can appear only with some event types that are atomic, but it cannot appear with other event types that are non-atomic. Before examining the supporting data for this similarity, a brief discussion on atomic and non-atomic events is in order. It is a

9) Nouns in Chinese may be classified into a more refined way than assumed in this paper, e.g., see Zhang (2013). For the parallel between CLn and CLv proposed in this paper, the coarse division (atomic vs. non-atomic) is sufficient to capture the parallel. 
well accepted view in the literature that an event can be characterized as being atomic or non-atomic on a par with a nominal that is atomic or non-atomic (e.g., Bach, 1986).10) Under this view, event classes - accomplishment, achievement, and semelfactive- are characterized as being atomic, while activity and state are characterized as being non-atomic. An event characterized as being atomic has a similar denotation to a nominal that is atomic, as discussed above: its minimal part is identifiable like a count noun such as 'pen'. In contrast, an event characterized as being non-atomic is similar to the denotation of a non-atomic noun such as a mass noun 'oil' that is lacking with an identifiable atomic part.11) This paper assumes the division of events into atomic and non-atomic as in these studies.

With this background on the division of events into atomic and non-atomic classes, let us examine the data of an event CLv that supports the current proposal in which an event CLv is in a parallel to an individual CLn with respect to a semantic selection relation. The relevant data is illustrated in (8). The classification of events in Mandarin with respect to individual verb phrases is following the detailed study in Deng (2013), which I assume without further argument. In (8), each of the event CLvs appears with atomic events: bian with accomplishment (8a), $c i$ with achievement (8b), and xia with semelfactive (8c). Note that each of these event CLvs can appear only with the event type as shown in (8); for example, the CLv xia in (8c) cannot appear with accomplishment such as in (8a) or achievement as in (8b) (see the examples in (14) in section 4 for further detail).

(8) a. Yanyang kao-le dangao san bian.

Yanyang bake-PERF cake three $\mathrm{CLv}$

'Yanyang baked the cake three times.'

(i.e., baking of the same cake three times)

b. Yanyang jin-le bangongshi san ci.
Yanyang enter-PERF office
'Yanyang entered the office three times.'

10) In Bach (1986), an each verbal predicate denotes a set of eventualities that can be expressed as one of five classes of an event assumed in this paper. For ease of exposition, I use the term event rather than eventuality.

11) This is a core idea on the division of an event into atomic or non-atomic one proposed in Bach (1986). For the purpose of the paper, I do not replicate the semantic detail on this idea. 

c. Yanyang jintian ke-le shi xia.
Yanyang today cough-PERF ten CLv
'Yanyang coughed ten times today.'

In contrast to the data in (8) above, the data in (9) below shows that an event CLv cannot appear with non-atomic events such as activity and state. For example, in (9a), the event of Yanyang's laughing belongs to activity, and it cannot be pluralized via the event CLv xia. The contrast between (8) and (9) shows that an event CLv such as in (8) can appear only with an atomic event.

(9) a. *Yanyang jintian xiao-le shi xia.

Yanyang today laugh-PERF ten CLv

'Yanyang laughed ten times today.'

b. *Yanyang zuotian gaoxing-le san bian.

Yanyang yesterday happy-PERF three CLv

'Yanyang was happy three times yesterday.'

In addition to the similarity in a semantic selection relation, an event CLv shows another similarity to an individual CLn: its unit of counting is also an atomic element, which may be obvious to readers by now. For instance, in (8c) above, the event CLv xia appears with the semelfactive event of coughing that is atomic. In particular, it pluralizes an atomic event, and its number of occurrences is indicated by the numeral shi 'ten': there were ten occurrences of coughing event. As for an event CLv, thus, the unit of counting is an atomic event denoted by the verb phrase.

This section shows that an event CLv and an individual CLn show a parallel in that they can appear with an atomic element and their units of counting are also atomic elements. The differences between the two CLs such as pluralizing an event and an entity respectively follow from their different lexical nature: a CLv is associated with the verbal domain and a CLn with the nominal domain.

\subsection{Parallel between occasion CLv and container measure CLn}

This section shows that there is a parallel between a container measure CLn and an occasion CLv similar to the one between an individual CLn and an event CLv 
shown in the previous section. As the name suggests, a container measure CLn indicates a unit of a container denoted by the CLn. A container measure CLn is unlike an individual CLn in that it does not show a semantic selection relation with the noun that it appears with. Consider the examples of a container measure CLn in (10). In (10), the container measure CLn xiang 'box' can appear with either an atomic noun $b i$ 'pen' (10a) or a non-atomic noun you 'oil' (10b).

$$
\begin{aligned}
& \text { (10) a. san xiang bi } \\
& \text { three CLn pen } \\
& \text { 'three boxes of pens' } \\
& \text { b. shi xiang you } \\
& \text { ten CLn oil } \\
& \text { 'ten boxes of oil' }
\end{aligned}
$$

Moreover, as these examples suggest, a container measure CLn counts the number of container denoted by the classifier, but not the number of the entity denoted by the noun, unlike an individual CLn. In (10), the nouns, regardless of whether it is atomic or non-atomic, are counted by the number of 'box', the unit indicated by the CLn xiang. For instance, the numeral shi 'ten' in (10a) indicates the number of a box, but is not associated with the number of a pen. It may be that each of three boxes contains several pens or merely one pen.

Turning to an occasion CLv, it shows a similar property to a container measure CLn: it does not show a semantic selection relation with the types of an event that it appears with. It can appear not only with atomic events such as accomplishment, achievement, or semelfactive, but also with non-atomic events such as activity or state that is not compatible with an event CLv (see (9)). This is exemplified with state (11a), activity (11b), and with semelfactive (11c) below.

(11) a. Yanyang jintian shangxin-le san hui. Yanyang today sad-PERF three CLv 'Yanyang was sad in three occasions today.'

b. Yanyang xiao-le san hui. Yanyan laugh-PERF three CLv

'Yanyang laughed on three occasions.' 
c. Yanyang qiao-le men san hui.

Yanyang knock-PERF door three CLv

'Yanyang knocked on the door in three occasions.'

An occasion CLv is also similar to a container measure CLn with respect to the unit of counting. An occasion CLv pluralizes an occasion in which the event takes place, namely the unit 'occasion' denoted by the CLv similarly to a container measure CLn. For example, in (11c), what is pluralized is the occasion indicated by the CLv hui: three occasions of the event of Yanyang's knocking on the door, which are temporally disjointed (see section 3.1). In the instance in (11c), it is not known how many times the event happened in each of the occasions: it may take place several times or just one time, which can be expressed by an event CLv. This is illustrated in (12) below. The first clause in (12) expresses that there was one occasion of the event indicated by the occasion CLv hui: one occasion of the event of her knocking on the door. The second clause in (12) expresses that this occasion consists of multiple events of her knocking on the door. In particular, the occasion consists of 'three' occurrences of the event as indicated by the event CLv xia along with the numeral. Thus, the unit of counting for an occasion CLv is not an individual event denoted by the VP but an occasion indicated by the CLv (hui), similar to a container measure CLn discussed above.

(12) Jintian xiawu, ta qiao-le men yi hui. this afternoon she knock-PERF door one CLv 'She knocked on the door in one occasion this afternoon.'

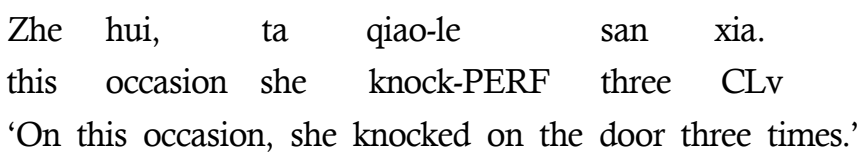

This section and the previous section have shown that an event CLv parallels to an individual CLn, and an occasion CLv to a container measure CLn as summarized in Table 1 below. In particular, the former pair pluralizes an atomic element, and the latter pair pluralizes the unit denoted by the CLs, i.e., an occasion and a container. 
Table 1 Summary of the parallels between the CLvs and the CLns

\begin{tabular}{ccc} 
Corresponding CLs & $\begin{array}{c}\text { Event CLv }- \\
\text { Ondividual CLn }\end{array}$ & $\begin{array}{c}\text { Ocasion CLv }{ }^{-} \\
\text {Container measure CLn }\end{array}$ \\
\hline \hline Unit of counting & $\begin{array}{c}\text { Atomic element } \\
\text { (an event }- \text { an entity) }\end{array}$ & $\begin{array}{c}\text { Unit denoted by the CLs } \\
\text { (an occasion - a container })\end{array}$ \\
Selection relation & Yes & No \\
\hline
\end{tabular}

Having established a parallel between the CLvs and CLns makes it possible to draw a parallel between event and nominal plurality in the language. An event pluralized by an event CLv is in parallel to a nominal pluralized by an individual CLn: both types of plurals are plurals of an atomic element denoted by the event and the nominal respectively. On the other hand, an event pluralized by an occasion CLv is in a parallel to a nominal pluralized by a container measure CLn: both types of plurals are plurals of the unit denoted by the each of the CLs.

\section{Consequences: Syntactic Parallel between a CLv and a CLn}

This paper proposed that a parallel between event and nominal plurality in Mandarin can be characterized in terms of the corresponding CLvs and CLns between the event and nominal domains, by building on the similar semantic properties of those CLvs and CLns. This aspect of the proposal predicts that there may be a certain syntactic parallel between the corresponding CLvs and CLns in accordance with their semantic similarities. ${ }^{12)}$ This section shows that this prediction is correct, as supported by the syntax of CLs proposed in current literature on Mandarin.

This paper has shown that both an individual CLn and an event CLv show a similar selection relation appearing with a particular semantic type of an event or a nominal. I propose that this type of the selection relation can be syntactically represented as a head and complement relation by building on the current Mandarin literature. In the nominal domain, an individual CLn type has been proposed to have such a structural representation (e.g., Cheng and Sybesma, 1998; Li, 2011;

12) A relevant question is to what extent such a parallel is possible. As shown in this section, the syntax of the CLs reflects the semantic selection relation of the CLs to some extent. At this stage, it is not clear whether more refined syntactic parallel could be drawn, which is beyond the scope of this paper. 
Zhang, 2013). As for an event CLv, a similar representation has been recently proposed (Zhang, 2017; Kim, 2019); furthermore, these recent studies proposed that a CLv and a CLn share the same core structure, as schematically illustrated in (13).13) In (13), the CL head can be realized as either a CLn or a CLv. In the previous studies, a type of a CLn has not been an issue, and the proposed structural similarity was rather a general one that includes both types of CLvs. Being more specific than the previous studies, I propose that the CL head in (13) represents either an event CLv or an individual CLn in a context where a relevant atomic element such as an event or a noun is pluralized. In a context where an atomic event denoted by a VP is pluralized, the head CL in (13) represents an event CLv that selects a VP complement. In a context where an atomic noun is pluralized, on the other hand, the CL head in (13) represents an individual CLn that selects a NP.

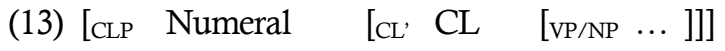

With an event CLv that realizes the CL head in (13), the semantic selection relation between an event $\mathrm{CLv}$ and its associated atomic event shown in section 3 can be read off from the head-complement syntax. Recall that there are the sets of classifiers in the language that can instantiate an event CLv but not an occasion CLv (and vice versa, see section 3). For example, the CLv xia or bian are event CLvs, and they cannot play a role of an occasion CLv such as hui that does not show a semantic selection relation. Thus, the head-complement structure as in (13) accounts for the distribution of event CLv. Moreover, the structure in (13) captures micro-differences among the event CLvs: in section 3, it is shown that a different event CLv appears with a specific class of an event. For instance, the event denoted by the VP has to be semelfactive when the CLv head is realized with xia as shown in (14a). Other event CLvs cannot replace the CLv xia; as shown in (14b), for example, the event CLv bian cannot appear with the semelfactive event in (14a) that appears with xia. As shown in section 3.2, the CLv bian (see (8a)) can appear with accomplishment only.

(14) a. Yanyang qiao-le $\quad$ men san xia.
Yanyang knock-PERF door three CLv
'Yanyang knocked on the door three times.'

13) In Zhang (2017), it is shown that a CLv is a head that takes a VP complement. See Zhang (2017) for evidence that a CLv is a verbal head. For the purpose of this paper, I do not replicate them. 

b. *Yanyang qiao-le men san bian. Yanyang knock-PERF door three CLv 'Yanyang knocked on the door three times.'

A similar pattern of the semantic relation is also observed with an individual CLn. As exemplified in (15a), the individual CLn zhi can appear with an atomic noun $b i$ 'pen'. However, another individual CLn $b u$ cannot appear with the same atomic noun 'pen', as shown in (15b). As illustrated in (15c), the CLn bu can appear with an atomic noun such as shu 'book'. These data shows that an individual CLn also shows a micro-difference in a semantic selection relation similar to an event CLn. The proposed head-complement syntax of an event CLv and an individual CLn such as in (13) captures the semantic selection relation shown by these CLs.

(15) a. liang zhi $\quad$ bi
two CLn
'two pens'
$\begin{array}{lll}\text { b. *liang } & \text { bu } & \text { bi } \\ \text { two } & \text { CLn } & \text { pen }\end{array}$

Intended meaning: 'two pens'

$\begin{array}{lll}\text { c. liang bu } & \text { shu } \\ \text { two } & \text { CLn } & \text { book } \\ \text { 'two books' } & \end{array}$

Turning to a container measure CLn and an occasion CLv, they have a commonality in that they do not show a semantic selection relation. In the current literature, a container measure CLn is viewed to be an adjunct to an NP (e.g., Li and Thomson, 1981; Tang, 1990; Li, 2011; Zhang, 2013), as schematically presented in (16). ${ }^{14)}$ In (16), a container measure CLn forms an adjunct CLnP along with a numeral (Nml), and appears as an adjunct to an NP. The lack of the semantic selection relation between the CLn and the noun naturally follows from the adjunct status of CLnP.

14) A common view of these studies is that a container measure CLn is originated as a $\mathrm{N}$ within a CLnP, which I do not question. 


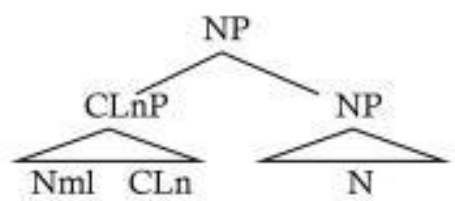

As for an occasion CLv, in some studies, it was proposed that an occasion CLv also has a head-complement structure similar to the one in (13) proposed for an event CLv (Zhang, 2017; Kim, 2019). For example, in Zhang (2017), a CL head realized by an occasion CLv selects a vP complement. However, the lack of the semantic selection relation shown by an occasion CLv suggests that it cannot have a head-complement structure unlike an event CLv and an individual CLn. Thus, I support another study such as Deng (2013) in which an occasion CLvP is an adjunct to the verbal projection, which is similar to the structure of a container measure CLn in (16).15) Under this type of an approach, the absence of semantic selection relation between an occasion CLv and an event that it appears with can be accounted for. For example, by building on Deng (2013), an adjunct analysis of an occasion CLvP can be schematically represented as in (17). In (17), CLvP appears as an adjunct to vP assuming that an occasion CLv appears above vP (e.g., Zhang, 2017; Kim, 2019).16) Being an adjunct to the vP that appears higher than the VP, an occasion CLv can appear with any classes of an event denoted by the VP.

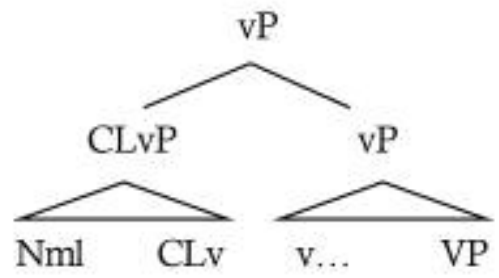

There is independent evidence that suggests that an adjunct view of an occasion CLv fares better than a head-complement approach. The relevant evidence comes

15) Although Deng (2013) proposed that an occasion CLv such as hui is an adjunct, no headcomplement structure of an even CLv has been suggested.

16) It may be the case that an occasion $C L v$ adjoins to a higher phrase than a vP being associated with temporality, given that it pluralizes a temporally disjointed event (see section 3.1). A precise adjunction position of an occasion CLv does not affect the main proposal of this paper. As this issue is beyond the scope of this paper, I leave it for future research. 
from VP idioms. In Mandarin, there are some idioms that consist of a verb and an object, as illustrated in (18). In (18), the verb tong 'poke' and the object mafeng-wo 'hornet-nest' together have an idiomatic meaning as well as a literal meaning as indicated in the gloss (Deng, 2013). The event denoted by the VP can be pluralized with an occasion CLv hui as in (18). In this case, both idiomatic and literal meanings are preserved. This fact supports the absence of a semantic selection relation of an occasion CLv: hui is compatible with either semelfactive event indicated by the literal meaning or accomplishment event indicated by the idiomatic meaning.

(18) ta tong-le mafeng-wo san hui.

s/he poke-PERF hornet-nest three CLv

(i) 'S/he poked a hornet-nest three occasions.'

(ii)'S/he offended someone not to be trifled with three occasions.'

(Deng, 2013)

In contrast, the same VP cannot appear with an event CLv such as xia when it indicates the idiomatic meaning. Rather, it is compatible with the event CLv xia only when it has the literal meaning, as shown in (19). As mentioned earlier, the event CLv xia appears with semelfactive event only. The idiomatic meaning is not semelfactive, and thus it cannot surface with the event CLv xia.

(19) ta tong-le mafeng-wo san xia.

s/he poke-PERF hornet-nest three CLv

(i) 'S/he poked a hornet-nest three times.'

(ii) *'S/he offended someone not to be trifled with three times.'

(Deng, 2013)

The difference that an occasion CLv shows from an event CLv with respect to VP idioms provide support for an adjunct view of the former and a headcomplement view of the latter.

The consequence of the discussion in this section suggests that the current syntax of CLn and CLv in the literature is in line with the proposed account on the semantic parallel between the CLvs and CLns in this paper. Moreover, it supports various forms of views in which there is a syntactic parallel between verbal and nominal domains generally (e.g., Abney, 1987; Megerdoomian, 2008 among many others). A common insight of these studies is that the locus of the parallel lies in 
a corresponding head between the two domains, which is further supported by the proposed account in this paper in which there are parallels between the corresponding CL heads between the two domains.

\section{Conclusion}

This paper shows that two types of CLvs in Mandarin correspond to two different CLns respectively, by building on the facts that the corresponding CLs show a similar semantic selection relation and their unit of counting is also similar. With the proposed correspondence, this paper further proposed that event and nominal plurality are in parallel in terms of the corresponding CLs.

The consequences of this paper not only contributes to the ongoing discussion on the event domain and its parallel to the nominal domain in the literature (e.g., Abney, 1987; Megerdoomian, 2008) with a particular focus on plurality, but it also suggests that event plurality is not a unified phenomena in a natural language similar to nominal plurality (e.g., Carson, 1977; Link, 1983; Rothstein, 2010) as the recent literature on event plurality has recognized (e.g., Henderson, 2013). Nominal plurality is much finer grained than a basic count-mass distinction; e.g., mass nouns do not form a uniform category, as the works on different semantics on a bare plural (Carlson, 1977; Zweig, 2009) suggests. The Mandarin case shown in this paper together with the languages that show mass reading of event plurality suggests that event plurality is non-uniform in terms of meaning (mass vs counting), but also in its forms (verbal plural marker vs verbal classifier).

\section{References}

Abney, S. P. (1987). The English noun phrase in its sentential aspect: Cambridge, MA: Massachusetts Institute of Technology.

Allan, K. (1977). Classifiers. Language, 53(2), 285-311.

Bach, E. (1986). The algebra of events. Linguistics and Philosophy, 9(1), 5-16.

Borer, H. (2005). Structuring sense, Vol. I: In name only. Oxford: Oxford University Press.

Cabredo Hofherr, P. (2010). Verbal plurality and event plurality. Lecture notes at the DGfS-CNRS Summer School on Linguistic Typology, Leipzig, 15 Aug-3 Sept.

Carlson, G. (1977). Reference to kinds in English (Doctoral dissertation). University of 
Massachusetts, Amherst.

Chao, Y. R. (1968). A grammar of spoken Chinese. California: University of California Press.

Cheng, L. L.-S., \& Sybesma, R. (1998). Yi-wan tang, Yi-ge tang: Classifiers and massifiers Tsing-Hua. Journal of Chinese Studies, 28(3), 385-412.

Chierchia, G. (1998). Plurality of mass nouns and the notion of "semantic parameter". In S. Rothstein (Ed.), Events and grammar (pp. 53-103). Dordrecht: Kluwer.

Corbett, G. G. (2000). Number. Oxford: Cambridge University Press.

Croft, W. (1994). Semantic universals in classifier systems. Word, 45, 145-171.

Cusic, D. D. (1981). Verbal plurality and aspect (Doctoral dissertation). Stanford University, California.

Deng, D. (2013). The syntax and semantics of event quantifiers in Mandarin Chinese (Doctoral dissertation). University of Wisconsin-Madison.

Greenberg, J. (1972). Numeral classifiers and substantival number: problems in the genesis of a linguistic type. Working Papers on Language Universals, 9, 2-39.

Henderson, R. (2012). Ways of pluralizing events (Doctoral dissertation). University of California, Santa Cruz.

Iljic, R. (2001). The problem of the suffix -men in Chinese grammar. Journal of Chinese Linguistics, 29(1), 11-68.

Jackendoff, R. (1991). Parts and boundaries. Cognition, 41(1), 9-45.

Kim, K., \& Melchin, P. (2018). On the complementary distribution of plurals and classifiers in East Asian classifier languages. Language and Linguistic Compass, 12(4), e12271.

Kim, K. (2019). Parallelism between nominal and verbal domains: Evidence from verbal classifiers. Linguistic Research, 36(2), 183-212.

Li, C. \& Thompson, S. (1981). Mandarin Chinese: A functional reference grammar. Berkeley: University of California Press.

$\mathrm{Li}, \mathrm{X}$. (2011). On the semantics of classifiers in Chinese (Doctoral dissertation). Bar-Ilan University, Israel.

Link, G. (1983). The logical analysis of plurals and mass terms: A lattice-theoretical approach. In P. Portner., \& H. P. Barbara (Eds.), Formal semantics - the essential readings (pp. 127-147). Oxford: Blackwell.

Megerdoomian, K. (2008). Parallel nominal and verbal projections. In R. C. O. Freidin, \& M. L. Zubizarreta (Eds.), Foundational issues in linguistic theory: Essays in honor of Jean-Roger Vergnaud (pp. 73-103). Cambridge, Mass: MIT Press.

Newman, P. (1990). Nominal and verbal plurality in Chadic. Dordrecht: Foris.

Rothstein, S. (2010). Counting and the mass/count distinction. Journal of Semantics, 27(3), 343-397.

Smith, C. S. (1991). The parameter of aspect. Dordrecht: Kluwer.

Tang, C.-C. J. (1990). Chinese phrase structure and the extended X'-theory (Doctoral dissertation). 
Cornell University, Ithaca, NY.

Travis, L. (2010). Inner aspect. Dordrecht: Kluwer Academic Press.

Verkuyl, H. J. (1972). On the compositional nature of the aspects. Dordrecht: Reidel.

Wood, E. (2007). The semantic typology of pluractionality (Doctoral dissertation). University of California, Berkeley.

Wu, Y., \& Bodomo, A. (2009). Classifiers $\neq$ Determiners. Linguistic Inquiry, 40, 487-503.

Vendler, Z. (1967). Verbs and times. In Z. Vendler (Ed.), Linguistics in philosophy (pp. 97-121). Ithaca, NY: Cornell University Press.

$\mathrm{Yu}$, A. C. (2003). Pluractionality in Chechen. Natural Language Semantics, 11(3), 289- 321.

Verkuyl, Henk J. (1972). On the compositional nature of the aspects. Dordrecht: Reidel.

Zhang, N. N. (2013). Classifier structures in Mandarin Chinese. Berlin: De Gruyter Mouton.

Zhang, N. N. (2017). The syntax of event internal and event external verbal classifiers.

Studia Linguistica, 71(3), 266-300.

Zhu, D. (1982). Yufa Jiangyi [Lectures on grammar]. Beijing: Shangwu Press.

Zweig, E. (2009). Number-neutral bare plurals and the multiplicity implicature. Linguistics and Philosophy, 32, 353-407.

\author{
Kyumin Kim \\ Professor \\ Department of English Language and Literature 298 \\ Chungbuk National University \\ Chungdae-ro 1, Seowon-gu Cheongjusi, 28644 Korea \\ E-mail: kukim@chungbuk.ac.kr
}

Received: October 21, 2020

Revised version received: December 12, 2020

Accepted: March 8, 2021 